\title{
A Novel Analogue Circuit for Controlling Prosthetic Hands
}

\author{
Eduardo Mangieri, Arash Ahmadi, Koushik Maharatna, Siti A Ahmad, Paul H Chappell \\ Electronic Systems and Devices \\ School of Electronics and Computer Science, University of Southampton, UK \\ Email: $\{$ em07r, aa5, km3, saa05r, phc $@$ @ecs.soton.ac.uk
}

\begin{abstract}
This paper presents a compact analogue circuit for the controlling of prosthetic hands. The circuit captures directly surface EMG signals as the input by which the user will be able to select different postures. The proposed circuit is able to work using only one EMG source targeting patients with different levels of amputation. It is also adaptable for different users with different EMG amplitude signals and the motion of each finger can be varied in the circuit even with the single EMG. Real captured EMG signals are applied to the design and simulation results demonstrate the capability of the circuit in processing EMG signals and controlling the prosthetic hand in an efficient way. The circuit is designed and implemented with $0.12 \mu \mathrm{m}$ CMOS technology and consumes $4 \mathrm{~mW}$ power for a set of sample postures.
\end{abstract} Hand

Index Terms - Analogue signal processing, EMG, Prosthetic

\section{INTRODUCTION}

$\mathrm{P}$ ROSTHETIC hands are designed to help people who have a partial upper limb amputation. The artificial hands are supposed to mimic the activities of the actual hand - e.g., user intended finger movements to change grips and grip an object - as closely as possible. In a real hand such activities are controlled by neuronal signals. The number of achievable operations is limited by current technology and the small number of electromyographic (EMG) signals used to control the prosthetic hand. It is also observed that in majority of the cases, the level of the upper limb's amputations may limit the number of available EMG signals [1], and thereby limiting the number of possible operations further.

Different approaches have been proposed to process the available EMG signals for controlling the motion of the fingers in a prosthetic hand [2-9]. In general, the processing is done in digital domain [2, 6, 7] and using a neural network [3-5, 8, 9]. These methods require access to multiple EMG signals which are available for lower level of amputation only. As the amputation level becomes higher, fewer EMG sources are available, in the worst case leading to a single EMG signal [1]. Additionally, in order to increase the efficiency of a prosthetic hand in terms of gripping and holding different objects, feedback signals from the sensors embedded in the finger tips need to be processed [10] in conjunction with the available EMG signals.
In principle, the EMG signals are wide spectrum analogue in nature and depending on several factors. Its actual shape and amplitude can vary considerably from time to time and from person to person $[1,11]$. Thus it appears to be more pragmatic to process these signals in analogue domain rather than in the digital domain. Additionally, recent research shows that processing naturally occurring signals in the analogue domain may result in a significant computational advantage compared to processing them in digital domain [12, 13].

Keeping these facts in mind, in this paper a novel analogue circuit is proposed for efficient control of prosthetic hands using EMG signal directly from its source. Although the circuit can be used for processing multiple EMG signals with minor modification; here the worst scenario is considered where a single EMG signal is available. Also a simple but effective methodology for controlling the hand postures using the single EMG signal is proposed. Additionally the circuit has the capability of controlling the grips more efficiently by processing the feedback signals from finger tip force and slippery sensors in combination with the input EMG signal as suggested in [10] and thereby providing precise controllability of individual finger's movement.

\section{SYSTEM OVERVIEW}

A general block diagram of the proposed system is shown in Fig.1. It is composed of standard electrodes, an EMG signal analyzer, an analogue state machine, a signal processing unit and a prosthetic hand with embedded sensors for the detection of slip and force activity. The functionalities of each of these units are described in this section.

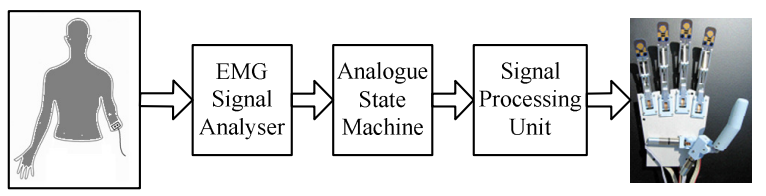

Fig. 1 Block diagram of the proposed system.

Using a single EMG signal for controlling different possible postures of a prosthetic hand leads to a "one to many" mapping problem and is extremely difficult to resolve. Even considering binary value of the EMG signal (' 1 ' = presence of EMG signal, ' 0 ' = no EMG signal) only 
two postures can be controlled. Here it is proposed a simple methodology to overcome this problem.

Each of the possible postures are associated with a "state". Transition from one "state" to another can be achieved by counting the number of successive EMG bursts if signal activity within a defined time window (T). As an example, to make a transition from state 1 to state 3 two EMG bursts have to be produced (physically, the user needs to stimulate his/her muscle two times) within the time $\mathrm{T}$. Generically, this process is described in Fig. 2, where three different hand postures are used as an example. The time window $\mathrm{T}$ is defined by the value of the signal "load cap". As long as "load cap" = 1 , we are within the specified time window $\mathrm{T}$. Thus, in principle, the entire control circuitry can be viewed as a simple state machine. The analogue design technique is used here for its implementation and thus in Fig. 1 it is referred as an analogue state machine. It is to be noted that the duration of the time window $\mathrm{T}$ may be adjusted from person to person depending on the output from their EMG amplifier. Table I describes the meaning of each posture associated with individual states. However the postures described in Table I are purely for example and any type of postures may be used corresponding to each state. The proposed methodology is scalable and can be adopted to incorporate more and complicated postures in the system, although here only three postures are considered.

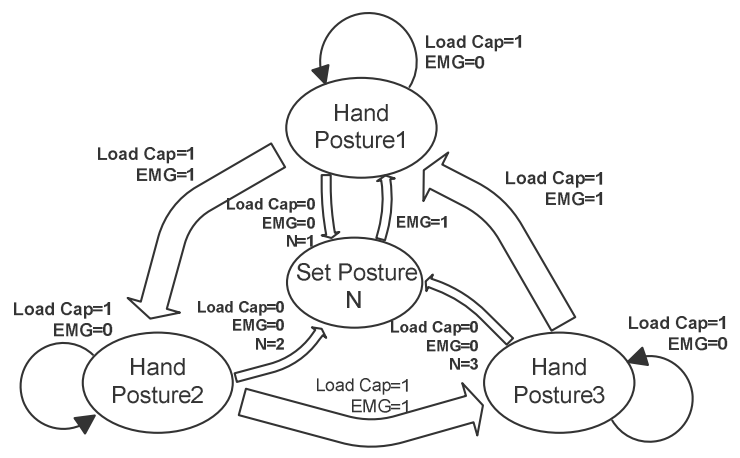

Fig. 2 State diagram of controlling different hand postures using single EMG signal

TABLE I: Example hand postures and number of related EMG signals.

\begin{tabular}{l|l|c} 
State & Posture & No. of EMG Stimuli \\
\hline Posture 1 & Open Hand & 1 \\
\hline Posture 2 & Lateral Grip & 2 \\
\hline Posture 3 & Spherical Grip & 3
\end{tabular}

Unfortunately the shape of the EMG signal does not lend itself as a suitable candidate for the above mentioned methodology. Thus, each EMG signal is converted to a square wave by a thresholding technique, as shown in Fig. 3 where a real life EMG signal has been used. This operation is done using the EMG analyzer circuit. For successive EMG signals the output of this circuit generates a pulse train where the number of the pulses in each sequence within the time window $\mathrm{T}$ is considered as an instruction to the analogue state machine to choose a particular posture from different predefined postures (and therefore the states) as described earlier.

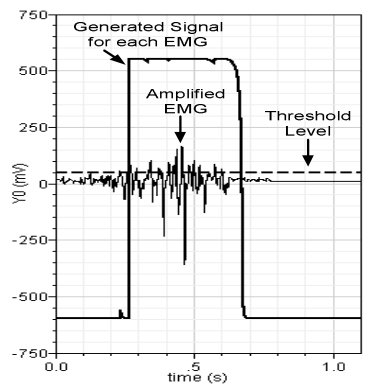

Fig. 3 Amplified EMG signal and generated square wave

After choosing a posture by the user, corresponding signals are sent to a signal processing unit, Fig. 4, to produce the required signals for controlling the motion of each finger.

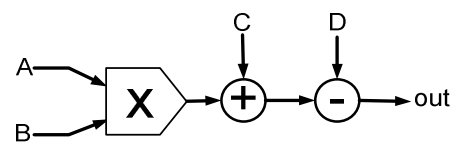

Fig. 4 Signal processing block diagram.

From a system level point of view, the signal processing unit is a general purpose analogue arithmetic unit, which is able to perform the first order multi-input polynomial operation as described in Eq. (1).

$$
\text { out }=(A \times B)+C-D
$$

Where, A is an input coming from the analogue state machine to control the motion of the prosthetic fingers; $\mathrm{B}$ is used as tuning input to vary the output voltage response. Input $\mathrm{C}$ and $\mathrm{D}$ are used for the control of slip and pressure feedback signals as they are summed and subtracted respectively to the input signals $(\mathrm{A} \times \mathrm{B})$.

\section{CIRCUIT IMPLEMENTATION}

To design the target system a $0.12 \mu \mathrm{m}$ technology and dual ended supply with value $\pm 0.6 \mathrm{~V}$ is used. A detailed explanation of the system sub-circuits, see Fig. 1, are presented in the following sub-sections.

A. EMG signal analyzer: Fig. 5 shows a block diagram and shape of the signal under processing at different nodes. The captured EMG signal shows amplitude of $200 \mu \mathrm{V}$ (peak to peak). The signal is amplified $1000 \times$ for ease of processing. The amplified signal has a low value $(\approx 0 \mathrm{~V})$ when a muscle is relaxed and a maximum of $100 \mathrm{mV}$ when contracted. Therefore, a comparator ( $\mathrm{C} 1$ in Fig. 5) with a reference voltage of $50 \mathrm{mV}$ is used to determine when the muscle is contracted (thresholding operation as mentioned in the previous Section) or not. The output of the comparator $\mathrm{C} 1$ is clamped by using a transistor configured as diode and a 50 $\mathrm{pF}$ capacitor. The clamped signal is compared to a reference voltage of $100 \mathrm{mV}$ using the comparator $\mathrm{C} 2$ for creating the desired square pulse which is used by the analogue state machine. Since small glitches can be generated by $\mathrm{C} 1$ each time the amplified EMG signal is near the reference voltage 
(50 mV) of $\mathrm{C} 1$, comparator $\mathrm{C} 2$ uses a higher reference voltage $(100 \mathrm{mV})$ to avoid further glitches at its output.

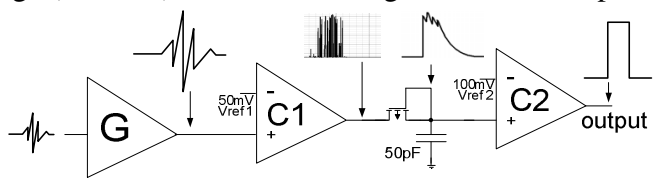

Fig. 5 EMG signal Analyzer block diagram.

B. The analogue state machine: Once the EMG signal is analyzed as shown in Fig. 5, the output of the comparator $\mathrm{C} 2$ is sent to the analogue state machine, of Fig. 6, to process the number of incoming EMG signals. Flip flops FF1 and FF2 are used as a 2-bit counter to keep track of the number of stimulated EMG signals; while the operational amplifier OP1 with the transistor M1 (set as a diode) and the capacitor Cap1 are configured as a peak detector.

This peak detector configuration can hold the output voltage for an amount of time equal to $1 \mathrm{~s}$ (the time window $\mathrm{T}$ mentioned in Section II). The user can send a successive EMG signals within this time window in order to change the desired posture of the prosthetic hand.z

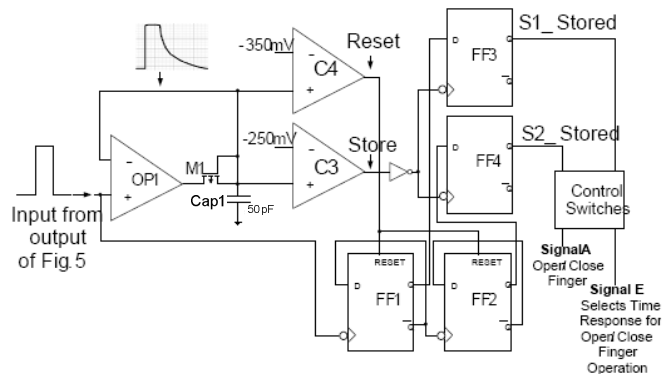

Fig. 6 The block diagram of the Analogue state machine

Comparators $\mathrm{C} 3$ and $\mathrm{C} 4$ are set to give $0.6 \mathrm{~V}$ at their output each time the clamped signal cross the reference voltages. The rising edge of the outputs of $\mathrm{C} 3$ and $\mathrm{C} 4$ are nearly concurrent, whereas the falling edges of them occur at 556 $\mathrm{ms}$ and $750 \mathrm{~ms}$ respectively. The output of C3 is used to store the values of the 2-bit counter into FF3 and FF4; while the output of $\mathrm{C} 4$ resets the counter. Therefore, the user has up to $556 \mathrm{~ms}$ to send a successive signal to switch to the next available grip. The control switch block shown in Fig.
6 , is used by the signals stored in FF3 and FF4 to control five analogue switches through which the actual operations of the hand is controlled.

C. The signal processing unit: Fig. 7 shows the transistor level diagram of a single signal processing unit, shown in Fig. 4. For controlling five fingers, five such units are employed in our design. The transistor dimensions used in this design are given in Table II. A standard multiplier core [14] with a first order RC component is used here. The circuit module shown in section (b) in Fig. 7 represents the current subtracting circuit used to process the output of the multiplier core in conjunction with the output currents of section (c) and (d). Section (c) and (d) rescale the amplitude of the slip and pressure feedback signals given at the nodes $\mathrm{C}$ and D respectively. Indeed, the current generated from section (c), in Fig. 7, is supplied to the positive input of the current subtracting section; whilst, the current generated from section (d), in Fig. 7, is connected to the negative input of the current subtracting circuit.

TABLE II: Transistor dimensions.

\begin{tabular}{c|c|c|c} 
Figure & Transistor & $(\mathbf{W} / \mathbf{L})$ & $\mathbf{W}(\boldsymbol{\mu m})$ \\
\hline $6 \mathrm{a}$ & $\mathrm{M}_{1-2}$ & 7.69 & 1 \\
\hline $6 \mathrm{a}$ & $\mathrm{M}_{3-6}$ & 30.76 & 4 \\
\hline $6 \mathrm{a}$ & $\mathrm{M}_{7-8}$ & 3.84 & 0.5 \\
\hline $6 \mathrm{a}$ & $\mathrm{M}_{9-11}$ & 3.46 & 0.45 \\
\hline $6 \mathrm{~b}$ & $\mathrm{M}_{1-6}$ & 1.15 & 0.15 \\
\hline $6 \mathrm{~b}$ & $\mathrm{M}_{7-8}$ & 1.92 & 0.25 \\
\hline $6 \mathrm{c}-\mathrm{d}$ & $\mathrm{M}_{1-2}$ & 3.69 & 0.48 \\
\hline $6 \mathrm{c}-\mathrm{d}$ & $\mathrm{M}_{3}$ & 23.07 & 3 \\
\hline $6 \mathrm{c}-\mathrm{d}$ & $\mathrm{M}_{4}$ & 15.38 & 2 \\
\hline $6 \mathrm{c}-\mathrm{d}$ & $\mathrm{M}_{5-6}$ & 1.15 & 0.15 \\
\hline $6 \mathrm{e}$ & $\mathrm{M}_{1-2}$ & 1.15 & 0.15 \\
\hline $6 \mathrm{e}$ & $\mathrm{M}_{3-4}$ & 307 & 40 \\
\hline $6 \mathrm{e}$ & $\mathrm{M}_{5-6}$ & 5 & 0.65 \\
\hline $6 \mathrm{e}$ & $\mathrm{M}_{7-8}$ & 3.84 & 0.5 \\
\hline $6 \mathrm{e}$ & $\mathrm{M}_{9}$ & 6.92 & 0.9 \\
\hline $6 \mathrm{e}$ & $\mathrm{M}_{10}$ & 15.38 & 2 \\
\hline $6 \mathrm{e}$ & $\mathrm{M}_{11-}$ & 1154 & 150
\end{tabular}

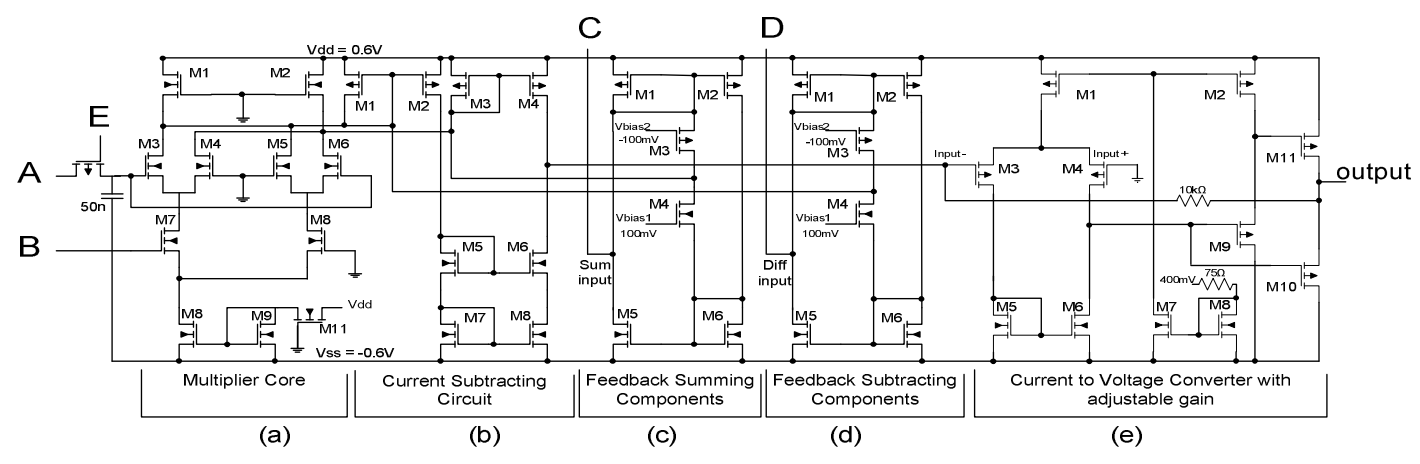

Fig. 7 Signal processing circuit schematic.

The Final stage of the signal processing unit is an adjustable gain current to voltage converter and the generated voltage is used for controlling the motors associated with each finger 


\section{Simulation Results}

In order to demonstrate the system performance, a simulation has been carried out using Cadence Spectre. Real life EMG signals are used for simulation purpose. However the repetition time of EMG pulses are varied in order to ensure the correct functionality of the entire system. After inputting the EMG signal into the system, the nature of processed signals are checked at the output of each units, as shown in Fig. 8-10 It is attainable from these graphs that the designed circuit has a correct functionality. Fig. 8 shows the captured EMG signal; the clamped signal with a discharging time of up to 1 second and the corresponding square pulse generated in the EMG analyzer circuit.

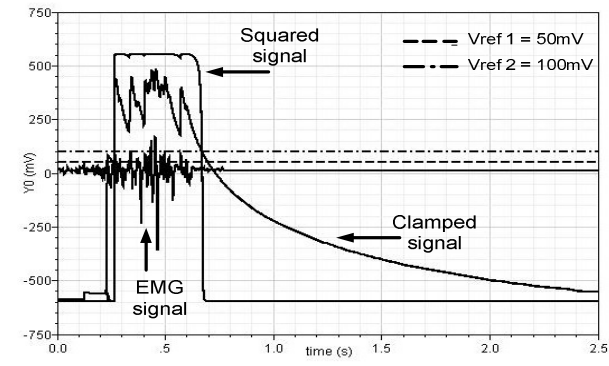

Fig. 8 Simulated internal signals of the EMG signal analyzer.

Fig 9 shows the circuit response of the analogue state machine when a set of EMG signals is applied to the system. Fig. 9a shows the square pulse generated by the EMG signal analyzer which is used as the inputs to the analogue state machine. Fig. 9b illustrates how the analogue state machine can hold the count and store the number of EMG pulses each time the interval between the successive EMG pulses exceed a predefined time (556 $\mathrm{ms}$ in this case).

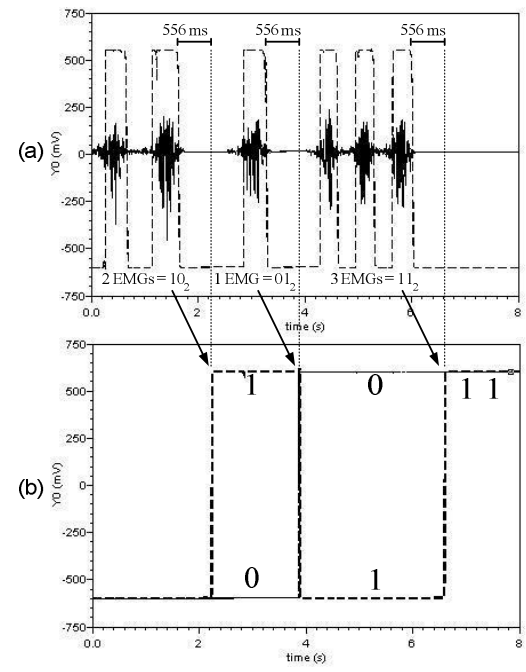

Fig. 9 Set of EMG signals and analogue state machine output response.

The most important of these is the result shown in Fig. 10 where the motions of the individual figures are depicted and response time of these figures can be varied. Current drawn from power supply is also measured for the postures, which shows $4 \mathrm{~mW}$ power consumption for the circuit overall.

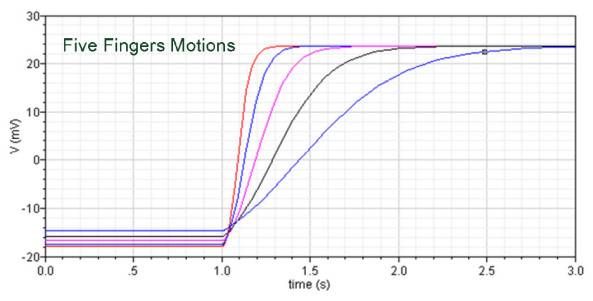

Fig. 10 Simulated responses for different fingers varying the RC constant (signal "E" of Fig. 7).

\section{DISCUSSION AND CONCLUSION}

In this work an analogue approach to the controlling of prosthetic hands with single EMG signals in combination with the feedback signals from slip and pressure sensors, which are embedded in the prosthetic hand, is presented. Using real surface EMG signals, the ability of the circuit to perform a configurable number of postures is demonstrated. It is also shown that time response of individual fingers can be varied using only single EMG in this circuit.

\section{REFERENCES}

[1] J. P. Derenne and W. A. Whitelaw, Acute respiratory Failure in Chronic obstructive Pulmonary disease: Marcel Dekker Ltd, 1996.

[2] D. Graupe, J. Magnussen, and A. A. Beex, "A Microprocessor System for Multifunctional Control of Upper-Limb Prostheses via Myoelectric Signal Identification," IEEE transaction on automatic control, vol. 23, pp. 538- 544, 1978.

[3] A. Hiraiwa, K. Shimohara, and Y. Tokunaga, "EMG Pattern Analysis and Classification by Neural Network," presented at IEEE International Conference on Systems, Man and Cybernetics, Cambridge, MA, USA, 1989, pp. 1113-1115.

[4] A. O. Andrade and A. B. SOARES, "EMG Pattern Recognition For Prosthesis Control," presented at COBEM 2001: Brazilian Congress of Mechanical Engineering, 2001, pp.

[5] M. F. Kelly, P. A. Parker, and R. N. Scott, "The application of neural networks to myoelectric signal analysis:a preliminary study," IEEE Transactions on Biomedical Engineering, vol. 37, pp. 221-230, 1990.

[6] Y. Konishi, Y. Mizobata, and M. Yoshida, "Development of a system for finding best electrode position for myoelectric hand control for derating of upper limb amputee," presented at EMBS 2007: 29th Annual International Conference of the IEEE, 2007 pp. $5699-5702$

[7] C.-L. Lin, S.-C. Wang, H.-C. Wu, S.-T. Young, M.-H. Lee, and T.-S Kuo, "A speech controlled artificial limb based on DSP chip," presented at EMBS 1998: 20th Annual International Conference of the IEEE, 1998 pp. 2704 - 2705.

[8] J. Zhao, Z. Xie, L. Jiang, H. Cai, H. Liu, and G. Hirzinger, "A Fivefingered Underactuated Prosthetic Hand Control Scheme," presented at BioRob 2006: The First IEEE/RAS-EMBS International Conference on Biomedical Robotics and Biomechatronics, 2006 pp. 995 - 1000.

[9] P. Zhou, M. M. Lowery, K. B. Englehart, H. Huang, G. Li, L. Hargrove, J. P. A. Dewald, and T. A. Kuiken, "Decoding a New Neural-Machine Interface for Control of Artificial Limbs," Journal of Neurophysiology, vol. 29, pp. 29, 2007.

[10] D. Cotton, P. H. Chappell, A. Cranny, N. M. White, and S. P. Beeby, "A Novel Thick-Film Piezoelectric Slip Sensor for a Prosthetic Hand," IEEE sensors journal, vol. 7, pp. 752 - 761, 2007.

[11] G. S. RASH, "Electromyography fundamentals," Available at: http://www.gcmas.org/EMGfundamentals.pdf. Accessed July 2008.

[12] J. Soler-Garrido, R. J. Piechocki, K. Maharatna, and D. McNamara, "Analog MIMO detection on the basis of Belief Propagation," presented at IEEE International Midwest Symposium on Circuits and Systems, 2006, pp. 50-54.

[13] "MIMO detection in analog VLSI," presented at IEEE International Symposium on Circuits and Systems, 2006 pp. 4.

[14] B. Razavi, Design of Analog CMOS Integrated Circuits, 1 ed: McGraw-Hill, 2000. 